\title{
Gastos de atención odontológica de niños con caries de infancia temprana, ocasionados a la familia y al Estado Peruano, representado por el Instituto Nacional de Salud del Niño
}

Costs of dental care of children with Early Childhood Caries, caused to the family and the Peruvian government, represented by the Child Heath National Institute

Gilmer Torres-Ramos ${ }^{1, a, c, f, f, g, k}$, Daniel José Blanco-Victorio ${ }^{2, a, d}$, Cynthia Anticona Huaynate ${ }^{3, a, g}$, Rosario RicseCisneros ${ }^{4, b, e, f}$, Virginia Antezana-Vargas ${ }^{5, a}$,

\section{RESUMEN}

Objetivo: Determinar los gastos de atención odontológica de niños con Caries de la Infancia Temprana (CIT) que ocasionan a sus familias y al Estado Peruano, representado por el Instituto Nacional de Salud del Nino (INSN) Lima-Perú. Material y Métodos: Estudio de tipo descriptivo, observacional y transversal. La muestra escogida aleatoriamente estuvo formada por 629 niños menores de 71 meses de edad atendidos en el Servicio de Atención del Infante del INSN, en el año 2009, dicha muestra fue estratificada según condición clínica ( sanos o con CIT) y procedimiento realizado (tratamiento preventivo y tratamiento restaurador: con manejo de conducta, sedación, o anestesia general). Para determinar los gastos ocasionados a las familias se entrevistaron a

Departamento de Odontología. Área de Odontopediatria. Instituto Nacional de Salud del Niño. Lima, Perú.

Facultad de Odontología Universidad Nacional Mayor de San Marcos. Lima, Perú.

Facultad de Odontología Universidad Nacional Mayor de San Marcos. Lima, Perú.

Departamento de Anestesiología del Hospital Angamos Essalud. Lima, Perú.

Facultad de Odontología Universidad Nacional Mayor de San Marcos. Lima, Perú.

Cirujano Dentista.

Médico Cirujano.

Especialista en Odontología Pediátrica.

Diplomado y Segunda Especialidad en Estadística en Investigación.

Especialista en Anestesiología.

Maestro en Gerencia de Servicios de Salud.

Doctora en Salud Pública y Epidemiología. 
las madres de los niños, y los gastos del hospital fueron calculados según el reporte económico del mismo. Para el análisis estadístico se utilizó el software Stata v12, aplicando las pruebas U. Mann-Whitney y Kruskal-Wallis para comparar y encontrar las diferencias en las variables estudiadas. Resultados: La frecuencia de CIT en la muestra evaluada fue de $82.19 \%$. Según mediana: las familias de pacientes sanos gastan por encima de S/.30.00 $(\min =12, \max =84)$ en procedimientos de prevención y las familias de los pacientes enfermos gastan por encima de $\mathrm{S} / .113 .00$ ( $\min =26, \max =761)$ en tratamientos restaurativos ocasionados por CIT, (Relación de 1:4). De acuerdo al tipo de tratamiento realizado, según la mediana, lo más resaltante se encontró en el rubro de anestesia general, donde las familias gastan por encima de $\mathrm{S} / .639 .00$ ( $\mathrm{min}=440, \max =761)$; es decir, 20 veces más que en procedimientos de prevención. Del gasto total, la mayor parte es asumida o subvencionada por el INSN, siendo estos gastos aún mayores. Conclusiones: La CIT, ocasiona importantes gastos directos e indirectos a las familias y al Estado representado por el INSN. Las medidas preventivas son altamente costo efectivas.

\section{PALABRAS CLAVE: Caries dental, economía, salud pública. (DeCS, BIREME)}

\section{SUMMARY}

Objective: To determine the cost of dental care for children with Early Childhood Caries (ECC) that causes to their families and the Peruvian State, represented by the National Institute of Child Health (INSN) LimaPeru. Methods: A descriptive, observation and transversal study. The randomly selected sample consisted of 629 children under 71 months of age, treated at the Infant Care Service of the INSN in 2009, the sample was stratified according to clinical condition (healthy or Early Childhood Caries) and procedure performed (preventive and restorative treatment: with behavior management, sedation or general anesthesia). To determine the costs incurred by families, mothers of children were asked and hospital costs were calculated according to the economic report. For statistical analysis software was used Stata v12, applying the Mann-Whitney U test and Kruskal-Wallis test to compare and find the differences in the studied variables. Results: The frequency of ECC in the evaluated sample was $82.19 \%$. According to the median, families of healthy patients spend over to $S / .30 .00$ $(\min =12, \max =84)$ in methods of prevention and families of sick patients spend over to $\mathrm{S} / .113 .00$ (min $=$ 26 , max $=761$ ) in restorative treatments caused by CIT, (ratio 1: 4). According the type of treatment performed, as reported by the median, most interesting observation was found under general anesthesia; families spend over to $S / .639 .00$ ( $\min =440, \max =761)$, ie 20 times more than in methods of prevention. The biggest part of total spending is taken or subsidized by the hospital, and these costs are even higher. Conclusions: ECC, cause significant direct and indirect costs to families and the State, represented by the hospital. Preventive measures are highly cost effective.

\section{KEYWORDS: Dental caries, economics, public health. (MeSH, NLM)}

\section{INTRODUCCIÓN}

La enfermedad más común de la infancia, es la Caries de Infancia Temprana (CIT). En la búsqueda de acceso a la atención y el tratamiento del dolor crónico y sus consecuencias, las familias experimentan estrés, la pérdida de ingresos por el tiempo dedicado llevar a un niño a múltiples citas con el dentista, el costo del transporte, etc. afectando a su economía y una disminución en la calidad de vida. Igualmente el Estado dedica recursos a la prevención y tratamiento de esta enfermedad. El sistema de salud se enfrenta a la gestión de las consecuencias extremas de la CIT en los servicios de urgencias hospitalarias y salas de operaciones $(1,2,3,4)$.

La CIT es una forma severa y particular de caries, muy virulenta y de carácter multifactorial, que afecta la dentición temporal de lactantes y niños pequeños en muchos países del mundo, comprometiendo por lo general numerosos dientes y produciendo una rápida destrucción e infección subsiguiente del tejido dentario $(5,6,7)$. La CIT es un término que describe sólo la caries dental en dientes primarios. Se ha afirmado que la CIT puede ser definida como la aparición de cualquier signo de lesión de caries 
dental en cualquier superficie del diente durante los 3 primeros años de vida (8). El término "Caries de la Infancia Temprana", fue adoptado por la Asociación Americana de Odontología Pediátrica para reflejar de mejor manera su etiología multifactorial e incluye la caries por biberón y la caries rampante (9). En el año 2003, la Academia Americana de Odontopediatría (AAPD) acordó la siguiente definición para la CIT: "La CIT es la presencia de una o más superficies cariadas (con o sin lesión cavitaria), superficies perdidas (debido a caries) o superficies obturadas en cualquier diente deciduo entre el nacimiento y los 71 meses de edad" $(10,11,12,13)$.

En la actualidad la CIT se considera un problema de salud pública, debido fundamentalmente a sus consecuencias sobre la salud de los niños y la economía de sus padres (14). El tratamiento de la CIT, es costoso porque la capacidad de cooperación de los bebés y niños en edad preescolar por lo general requiere el uso de anestesia general. Usualmente, el tratamiento consiste en la restauración o la extirpación quirúrgica de dientes cariados, junto con recomendaciones acerca de los hábitos de alimentación $(15,16)$. Si se deja sin tratamiento, puede generar abscesos, dolor y maloclusión; así como alteraciones en el crecimiento y desarrollo normal del niño, hospitalizaciones y atenciones de urgencia; con lo cual hay un deterioro en la calidad de vida de los niños $(6,8,10)$. Los niños que desarrollan caries de infancia temprana tienen riesgo de desarrollar procesos infecciosos como: celulitis, abscesos, osteomielitis, teniendo en cuenta que el desarrollo del proceso infeccioso es rápido. El dolor dental en casos avanzados lleva a incapacidad para alimentarse, por lo cual el niño tiende a perder peso $(15,17,18)$. Además la pérdida de alguno de los dientes temporales da como resultado, disminución de la función masticatoria, limitación en la cantidad de alimentos ingeridos y problemas para conciliar el sueño; dificultad para la fonación, problemas estéticos, etc., tanto antes como después de los tratamientos dentales $(9,12,15)$.

\section{MATERIAL Y METODOS}

El diseño de la investigación fue de tipo descriptivo, observacional y transversal. Se empleó el muestreo probabilístico, mediante una muestra aleatoria se seleccionó 629 niños atendidos en el Servicio de Atención del Infante del INSN, en el año 2009; con un margen de error de $2.2 \%$ y una confiabilidad del $95 \%$, posteriormente se estratifico la muestra para un mejor estudio, resultando: 112 niños sanos y 517 niños con CIT de los cuales: 92 niños fueron tratados con manejo de conducta, 238 niños con sedación y 187 niños con anestesia general, se incluyeron niños menores de 71 meses de edad, integrantes del programa SIS o pagante, no afectados por enfermedad sistémica y cuyas madres firmaron el consentimiento informado. Para determinar los gastos ocasionados a las familias se entrevistaron a las madres de los niños; y los gastos del hospital fueron calculados según el reporte económico del mismo. Para el análisis estadístico se utilizó el software Stata v12 (Stata Corporation, College Station, Texas), previamente los datos fueron sometidos a las pruebas de normalidad de Kolgomorov-Smirnov, no cumpliendo este supuesto se optaron por la pruebas no paramétricas, aplicando las pruebas $U$ de Mann-Whitney y Kruskal-Wallis, para comparar y encontrar las diferencias en las variables estudiadas. Los gastos de atención fueron calculados según tipo de tratamiento registrado por el hospital (costos en luz, agua, honorarios, equipos, etc.), los gastos ocasionados a las familias en la atención de los niños fueron declarados por la madre o acompañante del menor (pasajes, citas, alimentación, etc.) datos recogidos en fichas especialmente diseñadas, según objetivos del estudio.

\section{RESULTADOS}

Los pacientes sanos ambulatorios representaron el $17.81 \%$ y los pacientes enfermos con CIT representaron el $82.19 \%$. El mayor porcentaje de los niños enfermos, fueron tratados con sedación (37.84\%). Tabla 1.

Tabla 1. Distribución de los niños según condición clínica.

\begin{tabular}{lccc}
\hline \multicolumn{2}{c}{ Condición Clínica } & N & \% \\
\hline \multirow{2}{*}{ Sano } & & 112 & 17.81 \\
& Manejo de conducta & 92 & 14.64 \\
\multirow{2}{*}{ Enfermo } & Sedación & 238 & 37.84 \\
& Anestesia General & 187 & 29.73 \\
& Total & 629 & 100 \\
\hline
\end{tabular}


El gasto por tratamiento de CIT, ocasionado a las familias de los niños afectados, fue mayor en el tratamiento restaurador que en la prevención. Según mediana: el $50 \%$ de las familias de los pacientes sanos, gastan por encima de S/.30.00 en tratamientos de prevención y el $50 \%$ de las familias de los niños enfermos, gastan por encima de $\mathrm{S} / .113 .00$ en tratamientos restauradores ocasionados por la CIT (Tabla 2, Figura 1).

El costo asumido por el hospital en tratamientos restauradores de los niños afectados por CIT fue mayor que el costo asumido por procedimientos de prevención. Según la mediana: en el $50 \%$ de la muestra de niños sanos, el hospital gastó por encima de $\mathrm{S} / .260 .00$ y en el $50 \%$ de la muestra de niños enfermos, el hospital gastó por encima de S/.600.00 (Tabla 3, Figura 2).
El gasto total (gasto de familias + gasto hospital) de los tratamientos restauradores de los niños afectados por CIT es mayor que el costo total de los tratamientos de prevención. En el 50\% de la muestra de niños sanos se gastaron por encima de S/.290.00 en prevención, y en el $50 \%$ de la muestra de niños enfermos se gastaron por encima de S/.698.00 en tratamientos de restauración, ocasionados por CIT (Tabla 4, Figura 3).

El gasto del tratamiento de los niños afectados por CIT bajo anestesia general, según la mediana, fue mayor que el gasto por los tratamientos realizados con manejo de conducta o sedación. El $50 \%$ de la muestra de los sanos gastaron por encima de S/.30.00; mientras que el $50 \%$ de las familias de los niños enfermos atendidos con manejo de conducta gastaron por encima de S/.49.00; el mismo porcentaje de niños

Tabla 2. Gastos ocasionados por CIT, a las familias de los niños afectados, según condición clínica.

\begin{tabular}{lccccccc}
\hline $\begin{array}{c}\text { Condición } \\
\text { Clínica }\end{array}$ & Promedio & $\begin{array}{c}\text { Desviación } \\
\text { estándar }\end{array}$ & Mediana & RIQ & $\min$ & $\max$ & $\mathbf{p}$ \\
\hline Sano & 39.21 & 19.60 & 30 & 30 & 12 & 84 & $0.0000^{*}$ \\
Enfermo & 282.10 & 274.30 & 113 & 547 & 26 & 761 & \\
\hline
\end{tabular}

* $\mathrm{P}<0.05$ significativo (U de Mann-Whitney test)

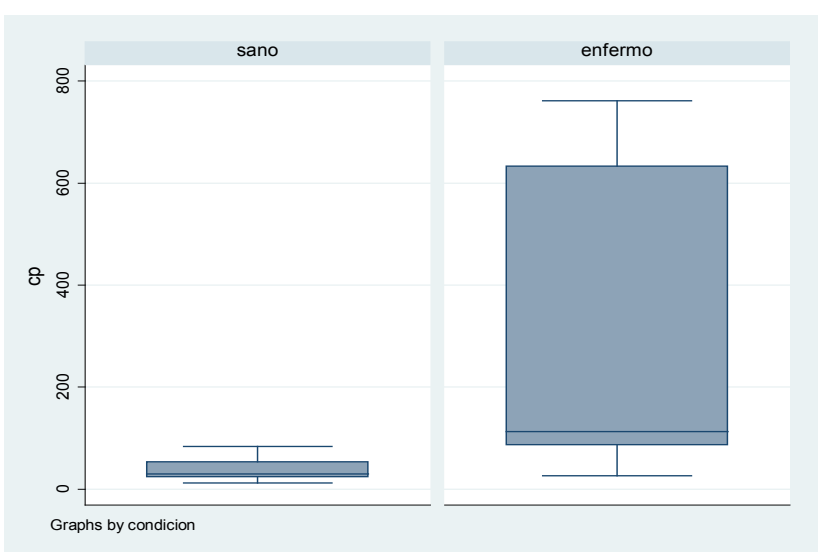

Figura 1. Gastos ocasionados por CIT, a las familias de los niños afectados, según condición clínica.

Tabla 3. Gastos por CIT asumidos por el hospital, según condición clínica de los niños.

\begin{tabular}{lccccccc}
\hline $\begin{array}{l}\text { Condición } \\
\text { Clínica }\end{array}$ & Promedio & $\begin{array}{c}\text { Desviación } \\
\text { estándar }\end{array}$ & Mediana & RIQ & min & max & P \\
\hline Sano & 310.36 & 139.03 & 260 & 200 & 80 & 620 & $0.0000^{*}$ \\
Enfermo & 1269.08 & 1095.57 & 600 & 2147 & 160 & 3486 & \\
\hline
\end{tabular}

$* \mathrm{P}<0.05$ significativo (U Mann-Whitney test) 


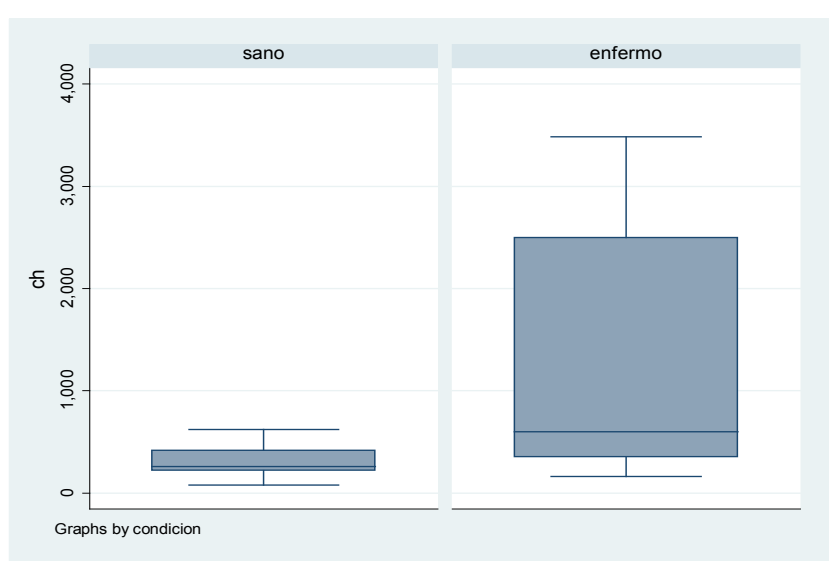

Figura 2. Gastos por CIT asumidos por el hospital, según condición clínica.

Tabla 4. Costo total del tratamiento en pacientes con CIT, según condición clínica.

\begin{tabular}{lccccccc}
\hline Condición Clínica & Promedio & $\begin{array}{c}\text { Desviación } \\
\text { estándar }\end{array}$ & Mediana & riq & min & $\max$ & P \\
\hline Sanos & 349.57 & 158.47 & 290 & 230 & 92 & 704 & \multirow{2}{*}{$0.0000^{*}$} \\
Enfermos & 1551.174 & 1364.47 & 698 & 2657 & 186 & 4108 & \\
\hline
\end{tabular}

*P $<0.05$ significativo (U Mann-Whitney test)

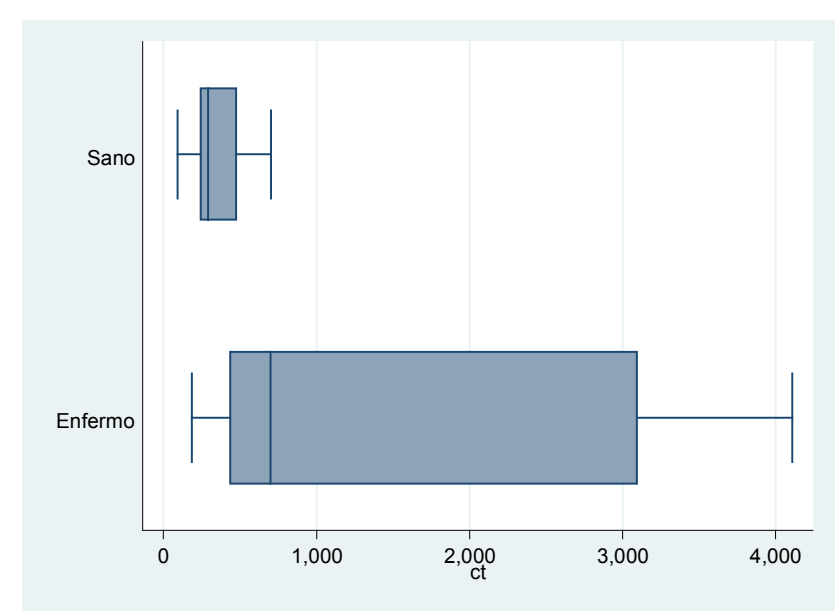

Figura 3. Costo total del tratamiento en pacientes con CIT, según condición clínica.

Tabla 5. Gastos ocasionados por CIT asumidos por las familias de los niños, según tratamiento clínico.

\begin{tabular}{lccccccc}
\hline Condición clínica & Promedio & D.E. & Mediana & RIQ & $\min$ & $\max$ & p \\
Sanos & 39.21 & 19.60 & 30 & 30 & 12 & 84 & \\
Enfermos & & & & & & & \\
\hline Manejo de conducta & 63.87 & 19.12 & 49 & 39 & 46 & 86 & $0.0001^{*}$ \\
Sedación & 87.58 & 33.19 & 98 & 60 & 26 & 150 & \\
Anestesia general & 637.03 & 91.92 & 639 & 80 & 414 & 761 & \\
\hline$* \mathrm{P}<0.05$ significativo (Kruskal-Wallis test) & & & & & &
\end{tabular}




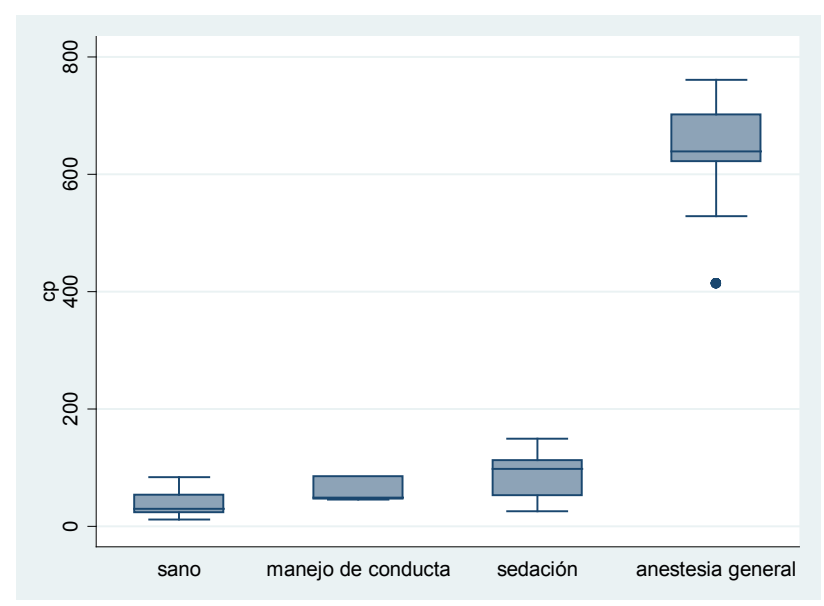

Figura 4. Gastos ocasionados por CIT asumidos por las familias de los niños, según tratamiento clínico.

Tabla 6. Gastos por CIT asumidos por las familias del paciente, según condición clínica: sano y enfermo, atendidos bajo anestesia general.

\begin{tabular}{lccccccc}
\hline \multicolumn{1}{c}{ Condición Clínica } & Promedio & D.E & Mediana & RIQ & min & max & p \\
\hline $\begin{array}{l}\text { Sanos } \\
\text { Enfermos }\end{array}$ & 39.21 & 19.59 & 30 & 30 & 12 & 84 & \\
Anestesia general & 637.03 & 91.92 & 639 & 80 & 414 & 761 & \\
\hline${ }^{*} \mathrm{P}<0.05$ significativo (U Mann-Whitney test) & & & & &
\end{tabular}

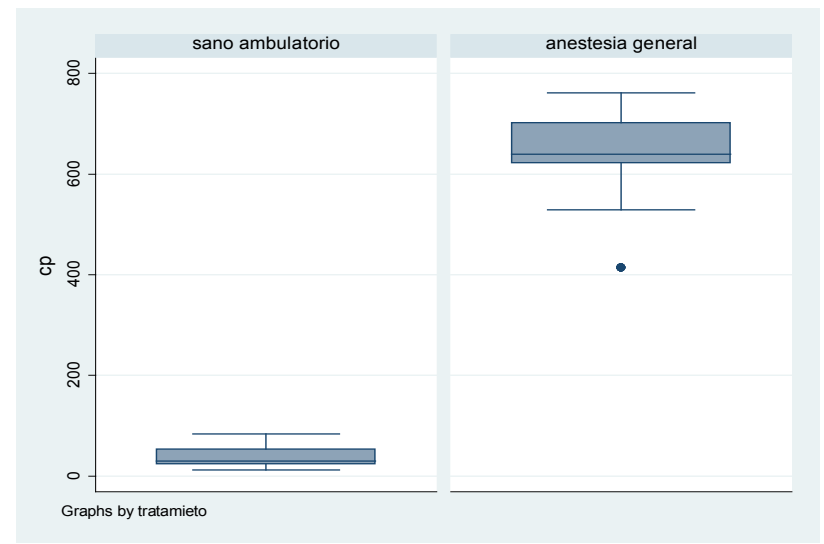

Figura 5. Gastos por CIT asumido por las familias de los pacientes según condición clínica: sano y enfermo, atendido bajo anestesia general.

atendidos bajo sedación gastaron $\mathrm{S} / .98 .00$ y el $50 \%$ de los pacientes atendidos con anestesia general gastaron por encima de $\mathrm{S} / .639 .00$. Estos gastos fueron asumidos por las familias de los pacientes atendidos. Encontrándose diferencias estadísticamente significativas $\mathrm{P}<0.05$ entre los grupos de pacientes (Tabla 5, Figura 4).
El gasto por el tratamiento restaurativo con anestesia general de los niños afectados por CIT, asumido por sus familias, es mayor que el gasto por tratamiento de prevención. En el 50\% de la muestra de los niños sanos las familias gastaron por encima de S/.30.00; y en el 50\% de la muestra de niños enfermos tratados con anestesia general, las familias gastaron 
por encima de $\mathrm{S} / .639 .00$, encontrándose diferencias estadísticamente significativas $\mathrm{P}<0.05$. Es decir las familias de pacientes enfermos tratados con anestesia general gastaron 20 veces más que los pacientes sanos (tratamiento de prevención), Tabla 6, Figura 5.

\section{DISCUSIÓN}

Se afirma que en salud, es mejor prevenir que curar y esto se evidencia cuando se analizan costos y gastos. El presente estudio permitió cuantificar el gasto ocasionado por la CIT, a las familias y al hospital que brindo la atención y el tratamiento.

Torres y col. (21) en el análisis de los datos sobre el impacto económico que generaba la caries de infancia temprana realizaron un análisis usando pruebas paramétricas. En el presente estudio se optó por pruebas no paramétricas al comprobar que los datos no cumplían el supuesto de normalidad, teniendo a la mediana como medida central de comparación, optimizando los resultados, donde se pudo apreciar una gran dispersión, en los gastos. Esto se explicaría porque el INSN, es un hospital de referencia nacional y los gastos se ven diferenciados. Muchas familias provienen de provincias y de los distritos más alejados del país, siendo los niños de las familias de escasos recursos los más vulnerables. El gasto que ocasiona la CIT a las familias de los niños afectados, es mayor en el tratamiento restaurativo que en el preventivo. Se encontró que esta diferencia es significativa mostrando una relación de 4:1 según la mediana de la muestra, resultados menores comparados a los hallados por Juárez y col. (19) quienes estudiaron necesidades de tratamientos dentales y análisis de costos odontológicos directos, en un grupo de pacientes con diabetes tipo 2, que fueron asistidos en un hospital argentino. Determinando que el costo de los tratamientos reparativos, era 15 veces más grande que el costo de la terapia preventiva. Coincidiendo en que las medidas preventivas son altamente costoefectivas, igualmente la gravedad de la afección. Tanto en la caries como en la diabetes, aumentan la necesidad de tratamientos más complejos acrecentando los recursos económicos necesarios para su resolución tanto para los familiares como para el Estado, en este caso, representado por el INSN.

Los resultados encontrados en el presente estudio son mayores a los encontrados por Squassi y col.
(20) quienes al evaluar un grupo de niños portadores $\mathrm{VIH}$, encontraron una relación de 1:3, comparando promedios de gastos de restauración en un programa preventivo. Estos autores concluyen que la atención con fuerte componente preventivo alcanza buena relación costo-beneficio para el control de caries dental en niños con infección por VIH.

Parte del gasto total de los tratamientos fueron subvencionados por el Estado, representado por el INSN. Este gasto se incrementa con la complejidad de la lesión y el uso de medidas más costosas para resolverlas como es el caso de tratamientos con anestesia general. Se pudo cuantificar resultando una relación de 1:10 siendo estos gastos aún mayores. En otras palabras, se estaría dejando de atender a 9 niños en tratamientos preventivos, por resolver un solo caso.

El conocimiento de las necesidades sociales a través de estudios de gastos que puedan ser ahorrados con medidas preventivas, es esencial en las directrices de estructuración, así como en la formación los recursos humanos y la distribución de los fondos destinados a la eficacia y la calidad en el sistema de salud, estos resultados nos permiten mostrar la importancia de la prevención. Las familias de escasos recursos son las más vulnerables, ya que el destinar recursos para resolver afecciones bucales afecta a su precaria economía. Este estudio cuantificó que, en caso que se requiera atención restaurativa de casos complejos con anestesia general, comparado con medidas preventivas, la relación del gasto es de 1:21. Es necesario remarcar la importancia de un programa de impacto sustancial de medidas preventivas.

En conclusión, la CIT como problema de salud pública, ocasiona importantes gastos directos $\mathrm{e}$ indirectos a las familias y al Estado representado por el INSN. Las medidas preventivas son altamente costo-efectivas.

\section{Correspondencia}

\section{Gilmer Torres Ramos}

Av. Olavegoya 2017, Jesús María. Lima, Perú. Correo electrónico: gilmertorres1974@gmail.com 


\section{REFERENCIAS BIBLIOGRÁFICAS}

1. Casamassimo PS, Thikkurissy S, Edelstein BL, Maiorini E. Beyond the dmft: The human and economic cost of early childhood caries. J Am Dent Assoc. 2009; 140(6):650-7.

2. Bernabé E, Delgado E, Sánchez P. Resultados de un sistema para la vigilancia de caries de la infancia temprana. Rev Med Hered. 2006; 17(4):227-33.

3. Cravero JP, Blike GT, Beach M, Gallagher SM, Hertzog $\mathrm{JH}$, Havidich JE. et al. Incidence and nature of adverse events during pediatric sedation/anesthesia for procedures outside the operating room: report from the Pediatric Sedation Consortium. Pediatrics 2006;118(3):1087-1096.

4. Griffin SO, Gooch BF, Beltrán E, Sutherland JN, Barsley R. Dental services, costs, and factors associated with hospitalization for Medicaid-eligible children, Louisiana 1996-97. J Public Health Dent 2000;60(1):2127.

5. Horowitz HS. Research issues in early childhood caries. Community Dent Oral Epidemiol. 1998; 26(1):67-81.

6. Zaror C, Pineda P, Orellana JJ. Prevalencia de caries temprana de la Infancia y sus factores asociados en niños chilenos de 2 y 4 años. Int J Odontostomat. 2011; 5(1):171-7.

7. Gudiño S. Caries de la temprana infancia: denominación, definición de caso y prevalencia en algunos países del mundo. Pub Cient Fac Odont UCR. 2006; 1 (8):39-45.

8. Vachirarojpisan $\mathrm{T}$, Shinada $\mathrm{K}$, Kawaguchi Y, Laungwechakan P, Somkote T, Detsomboonrat P. Early childhood caries in children aged 6-19 months. Community Dent Oral Epidemiol. 2004; 32(2):133-42.

9. Babu J, Nigel M. Early childhood caries lesions in preschool children in Kerala, India. Pediatr Dent. 2003; 25(6):594-600.

10. Guerrero MP, Galeana MG, Corona AA. Caries de la infancia temprana: Medidas preventivas y rehabilitación. Rev Odontol Latinoam. 2011; 4(1):258.
11. American Academy of Pediatric Dentistry (AAPD). Oral Health Polices and Clinical Guidelines. Pediatr Dent. 2003; 25(7):9.

12. Montero D, López P, Castrejón RC. Prevalencia de caries de la infancia temprana y nivel socioeconómico familiar. Rev Odontol Mex. 2011; 15(2): 96-102.

13. Arango MC, Baena GP. Caries de la infancia temprana y factores de riesgo. Revisión de la Literatura. Rev Estomat. 2004; 12(1):59-65.

14. Alonso MJ, Karakowsky L. Caries de la infancia temprana. Perinatol Reprod Hum. 2009; 23 (2): 90-7.

15. Villena R, Pachas F, Sánchez Y, Carrasco M. Prevalencia de caries de infancia temprana en niños menores de 6 años de edad, residentes en poblados urbano marginales de Lima Norte. Rev Estomatol Herediana. 2011; 21(2):79-86.

16. Berkowitz RJ. Causes, treatment and prevention of early childhood caries: A microbiologic perspective. J Can Dent Assoc. 2003;69(5):304-7

17. American Academy of Pediatric Dentistry. Guideline on infant oral health care. Pediatr. Dent. 2012; 34(6):132-136.

18. Low W. The effect of severe caries on quality of life in young children. Pediatr Dent 1999; 21(6): 325 - 326.

19. Juárez R, Mazzáfero V, Gorodner V. Impacto económico de la hiperglucemia en la salud bucal de pacientes diabéticos tipo 2. Acta Odont Ven. 2009; 47 (1):1-13.

20. Squassi AF, Allesandrello H, D'Eramo L, Piovano S, Bordoni NE. Estudio de costos de la atención odontológica en niños afectados por VIH o con SIDA Bol Asoc Arg Odontol Niños. 2005; 34(3):12-18.

21. Torres G, Loayza R, Ricse R, Rivas P. Impacto Económico de niños que presentan caries de infancia temprana atendidos en el servicio de atención del infante del Instituto Nacional de Salud del Niño, LimaPerú 2009. Odontol Pediatr. 2013; 12(1):14-9.

Recibido: $25 / 11 / 2014$

Aceptado: 20/02/2015 